\title{
PERFORMANCE EVALUATION OF PROPRIOCEPTIVE SENSORS FOR COMPACTOR LOCALISATION
}

\author{
Denis Bouvet, Gaëtan Garcia \\ Institut de Recherche en Cybernétique de Nantes (IRCyN) UMR 6597 \\ 1 rue de la Noë, BP 92101, 44321 Nantes Cedex 03, France \\ E-mail: Denis.Bouvet@ircyn.ec-nantes.fr, \\ Gaetan.Garcia@ircyn.ec-nantes.fr
}

\begin{abstract}
This paper presents several localisation algorithms based on the Extended Kalman Filter approach and compares their respective performances. The main interest lies in the proposal of two different instrumentations able to replace odometry, unusable on work sites. Results are presented using data obtained on trials carried out at the Centre d'Expérimentations Routières (CER) in Rouen in collaboration with the Laboratoire Central des Ponts et Chaussées (LCPC).
\end{abstract}

Keywords: 2D Localisation, Extendēd Kalman Filter (EKF), Multisensory Data Fusion, Mobile Robots, Articulated Vehicles.

\section{INTRODUCTION}

Improving the global efficiency of road constructions requires among others the development of precision systems for the real-time localisation of civil-engineering vehicles [2]. For surfacing machines, such as compactors, the centimetre accuracy of the Real Time Kinematic (RTK) GPS is sufficient [5], since the $2 \mathrm{D}$ localisation tolerance is of a few decimeters. Yet, we cannot envisage the use of GPS technology alone, and this for two essential reasons:

- The average working speed of a compactor is $2 \mathrm{~m} / \mathrm{s}$ and the GPS signal frequency is 1 $\mathrm{Hz}$. Therefore, if we want to respect the positioning tolerance, we need further information about the machine evolution to evaluate the compactor position between two GPS measures.

- By tracking five or more satellites, the RTK surveying techniques can provide centimetre accuracy. But in the proximity of obstacles such as buildings or bridges, the line of sight from the receiver to some of the satellites can be blocked and the GPS data accuracy considerably degraded or unavailable. In these conditions, we cannot but have recourse to dead-reckoning navigation with proprioceptive measures.

The Kalman filtering approach allows us to fuse the GPS localisation with proprioceptive data. For indoor mobile robots, the most widely used method to provide the latter is odometry, but in the case of civil-engineering vehicles, this technique is inapplicable, for the required equipment

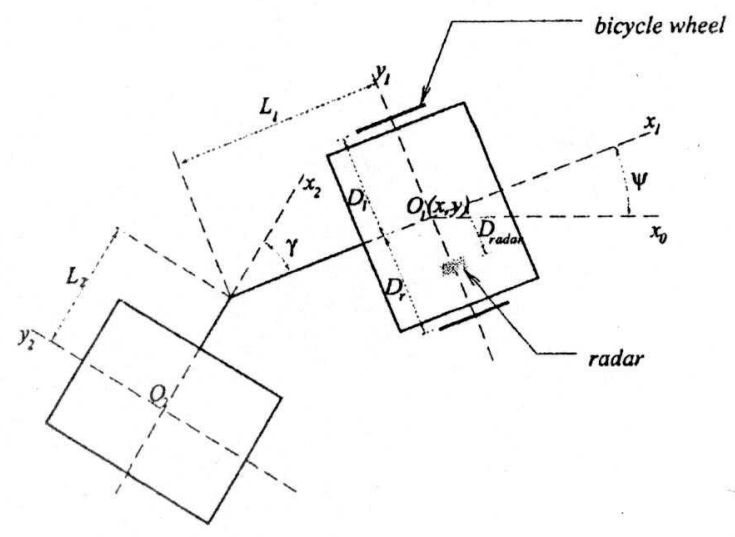

Figure 1: Compactor modelling (for more details, see [3])

is cumbersome and not robust enough. After the presentation of the EKF algorithm in section 2, we will study in section 3 two alternatives to using odometry and give the experimental results in section 4. Eventually, the conclusions are presented in section 5 .

\section{THE EXTENDED KALMAN FILTER AND THE RESULTING ALGORITHM}

The compactor is an articulated vehicle, which means that it has a front and a rear body which can rotate relative to each other. In the following discussion, the pose of the compactor will refer to the $(x, y)$ position of the centre of the front roll and the orientation $(\psi)$ of the front body relative to the work site frame $\left(R_{0}\right)$ (see figure 1$)$. The 
resulting state vector at the discrete time instant $i$ is given by $X_{i}=\left[\begin{array}{lll}x_{i} & y_{i} & \psi_{i}\end{array}\right]^{T}$.

Thanks to the proprioceptive measures $U_{i}$, which will vary with the sensor configuration, we can express the elementary translation $\Delta_{i}$ and rotation $\omega_{i}$ of the front roll. Assuming there is no lateral slip, we obtain the well-known kinematic evolution model,

$$
\left\{\begin{aligned}
x_{i+1} & =x_{i}+\Delta_{i}\left(U_{i}\right) \cos \left(\psi_{i}\right) \\
y_{i+1} & =y_{i}+\Delta_{i}\left(U_{i}\right) \sin \left(\psi_{i}\right) \\
\psi_{i+1} & =\psi_{i}+\omega_{i}\left(U_{i}\right)
\end{aligned}\right.
$$

which can be summarized by the following vector equation:

$$
X_{i+1}=F\left(X_{i}, U_{i}\right)
$$

The observation equation is even simpler, since the GPS antenna is situated above the centre of the front roll. Thus, we have at the observation instant $j$ :

$$
Z_{j}=\left[\begin{array}{c}
x_{j} \\
y_{j}
\end{array}\right]=G\left(X_{j}\right)
$$

\section{Remark 2.1}

We use a different notation for the observation time index because, in the following, equation (2) will be used at higher frequency than (3). Hence, (3) is not applicable at each time index $i$.

Actually, equations (2) and (3) are corrupted by noises which are supposed to be zero-mean, white, independent of each other and gaussian. The resulting system is given by:

$$
\left\{\begin{aligned}
X_{i+1} & =F\left(X_{i}, U_{i}^{*}\right)+\alpha_{i} \\
U_{i}^{*} & =U_{i}+v_{i} \\
Z_{j} & =G\left(X_{j}\right)+w_{j}
\end{aligned}\right.
$$

where

- $\alpha_{i}$ (covariance matrix: $Q_{\alpha}$ ) is the model noise which will account for the inaccuracy of (1) due to the restrictive hypothesis made to write the roll evolution equations,

- $v_{i}$ (covariance matrix: $Q_{v}$ ) is the noise which affects proprioceptive measures,

- $w_{j}$ (covariance matrix: $Q_{w}$ ) is the noise which affects RTK GPS measures.

\section{Definition 2.2}

The estimation of the state $X_{k}$ is denoted $\hat{X}_{k / l}$, which means the estimation of $X_{k}$ knowing what happened until instant $l$.

The associated covariance matrix is denoted : $P_{k / l}=E\left(\left(\hat{X}_{k / l}-X_{k}\right)\left(\hat{X}_{k / l}-X_{k}\right)^{T}\right)$
In order to estimate the state $X$, we use an EKF which requires to linearize the state-space description (4) around the current estimation $\hat{X}_{i / i}$ by calculating the following jacobian matrices:

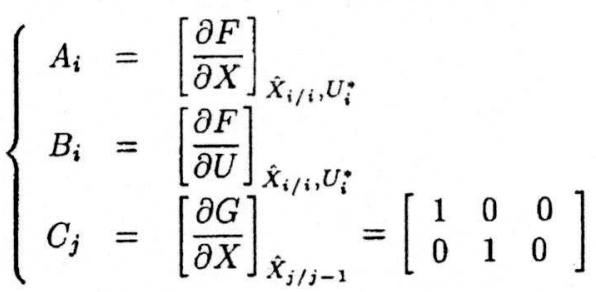

The resulting estimator works in two steps: prediction and filtering.

Between two RTK GPS measures, the state at time instant $i+1$ is predicted from the previous state $\hat{X}_{i / i}$ and the associated covariance matrix is calculated. The classical EKF equations are:

$$
\left\{\begin{aligned}
\hat{X}_{i+1 / i} & =F\left(\hat{X}_{i / i}, U_{i}^{*}\right) \\
P_{i+1 / i} & =A_{i} P_{i / i} A_{i}^{T}+B_{i} Q_{v} B_{i}^{T}+Q_{\alpha}
\end{aligned}\right.
$$

When a GPS measure occurs at instant $j$, the pose is corrected: this is the filtering step.

$$
\left\{\begin{aligned}
\hat{X}_{j / j} & =\hat{X}_{j / j-1}+K_{j}\left(Z_{j}-G\left(\hat{X}_{j / j-1}\right)\right) \\
P_{j / j} & =\left(I-K_{j} G\left(\hat{X}_{j / j-1}\right)\right) P_{j / j-1}
\end{aligned}\right.
$$

where $K_{j}$, the Kalman gain, is calculated by:

$$
K_{j}=P_{j / j-1} C_{j}^{T}\left(C_{j} P_{j / j-1} C_{j}^{T}+Q_{w}\right)^{-1}
$$

And the resulting algorithm gives:

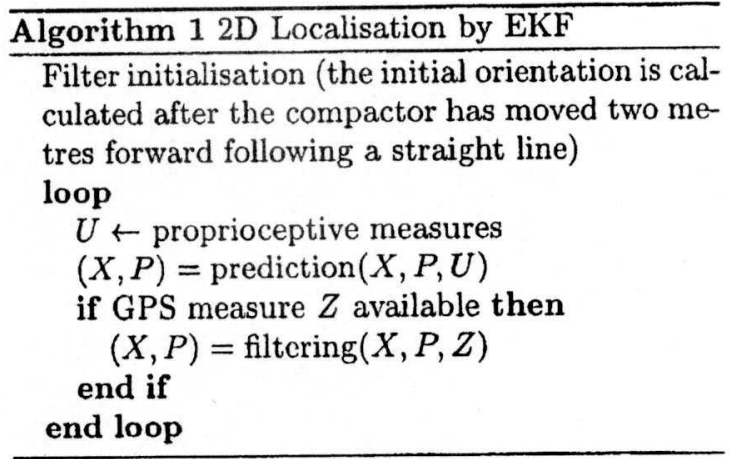

\section{DIFFERENT SETS OF PROPRIOCEPTIVE SENSORS}

\subsection{Encoder wheels}

A free bicycle wheel equipped with a precision encoder ( $4 \times 4096$ points per turn) is fixed on each side of the front roll. The resulting data are two rotation angles, $\theta_{l}$ and $\theta_{r}$, and the proprioceptive measures are given by:

$$
U_{i}=\left[\begin{array}{cc}
\Delta \theta_{l_{i}} & \Delta \theta_{r_{i}}
\end{array}\right]^{T}
$$




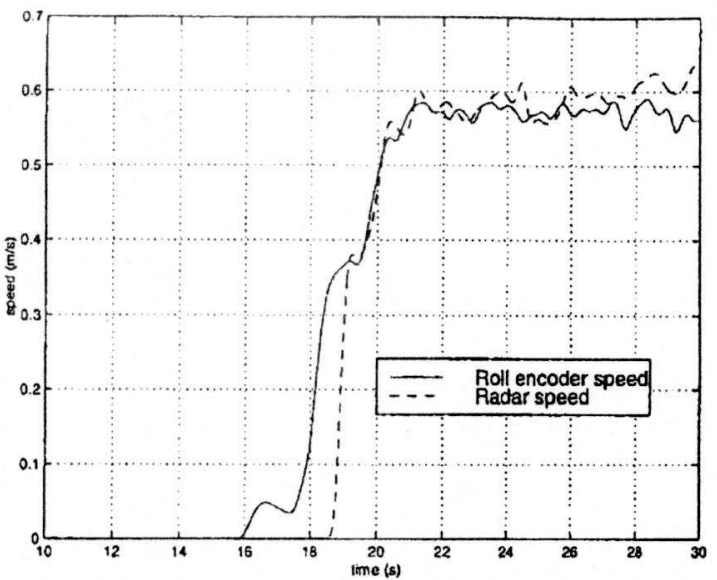

Figure 2: Comparison between the radar speed and the one deduced from the encoder measure $\theta$

Knowing the wheel radii $R_{l}$ and $R_{r}$, and the distance from the encoder to the roll centre $D_{l}$ and $D_{r}$, the elementary rotation and translation satisfy the following equation:

$$
\left[\begin{array}{c}
\Delta_{i} \\
\omega_{i}
\end{array}\right]=\left[\begin{array}{cc}
\frac{D_{r} R_{l}}{D_{l}+D_{r}} & \frac{D_{l} R_{r}}{D_{l}+D_{r}} \\
-\frac{R_{l}}{D_{l}+D_{r}} & \frac{R_{r}}{D_{l}+D_{r}}
\end{array}\right] U_{i}
$$

The results given by odometry are among the best we can expect in sheer dead-reckoning navigation. That is why we will consider them as a reference for the two other Kalman estimators, which are based on different proprioceptive sensors.

\subsection{Radar and gyrometre}

At the present time, this is the configuration chosen by the partners of the Brite-EuRarn CIRC project [4]. For our trials, the elementary rotation is given by a gyrometre mounted on the driver's cabin. For the elementary translation, two sensors are necessary: an encoder on the front roll, and a radar fixed at the front of the compactor.

The encoder gives the direction of the movement (forward or backward) and an estimation of the elementary translation at low speed. Indeed, as shown on figure 2 (for more clarity, a low-pass filter was applied to the data), the chosen radar is inefficient under $v_{\min }=0.4 \mathrm{~m} / \mathrm{s}$. Above this minimum limit, we use radar information, for this sensor is less affected by vibrations which can make the rolls take off and the encoder data valueless.

To sum up, at instant $i$, the proprioceptive measures are given by:

$$
U_{i}=\left[\begin{array}{lll}
V_{\text {radar }_{i}} & \Delta \theta_{i} & \omega_{g y r_{i}}
\end{array}\right]^{T}
$$

Since the radar is not situated on the axis $x_{1}$ (see fig. 1), we must take into account the distance from the centre of the front roll to the radar along the axis $y_{1}$. Let us denote $D_{\text {radar }}$ this algebraic distance, $R_{1}$ the radius of the front roll and $T_{s}$ the sampling period. The elementary rotation and translation write as follows:

$$
\left\{\begin{aligned}
\Delta_{i} & =R_{1} \Delta \theta_{i} \text { if } R_{1} \Delta \theta_{i}<V_{\min } T_{s} \\
& =\left(V_{\text {radar }_{i}}+D_{\text {radar }} \omega_{g y r_{i}}\right) T_{s} \text { if not } \\
\omega_{i} & =\omega_{g y r_{i}} T_{s}
\end{aligned}\right.
$$

Trials have already shown that the radar plus gyrometre configuration gave satisfying results. But the gyrometre is a costly instrument which sometimes has drift problems [1]. In the following section, a new set of sensors is proposed to replace it.

\subsection{Radar and steering angle}

The two equipment configurations we addressed were not specific to articulated vehicles. We purpose now to take into account this particularity of the compactor.

\section{Definition 3.1}

We call $\overrightarrow{V_{i j}}(P)$ the speed of a point $P$ belonging to the body $j$ in its movement relative to the reference frame $\left(R_{i}\right)$.

Let $\overrightarrow{V_{01}}\left(O_{1}\right)$ (resp. $\left.\overrightarrow{V_{02}}\left(O_{2}\right)\right)$ be the speed of $O_{1}$ (resp. $\mathrm{O}_{2}$ ), the centre of the front roll (resp. rear roll) relative to the work site frame. We get:

$$
\left\{\begin{array}{l}
\overrightarrow{V_{01}}\left(O_{1}\right)=V_{1 x} \overrightarrow{x_{1}}+V_{1 y} \overrightarrow{y_{1}} \\
\overrightarrow{V_{02}}\left(O_{2}\right)=V_{2 x} \overrightarrow{x_{2}}+V_{2 y} \overrightarrow{y_{2}}
\end{array}\right.
$$

Let $\gamma$ be the steering angle between the front and the rear body. Let $L_{1}$ (resp. $L_{2}$ ) be the distance between the articulation joint and $O_{1}$ (resp. $O_{2}$ ) (see fig. 1).

Provided $\gamma \neq 0$, lateral slip speeds write as follows:

$$
\left\{\begin{array}{l}
V_{1 y}=\frac{V_{2 x}-V_{1 x} \cos (\gamma)}{\sin (\gamma)}+L_{1} \dot{\psi} \\
V_{2 y}=-\frac{V_{1 x}-V_{2 x} \cos (\gamma)}{\sin (\gamma)}-L_{2}(\dot{\psi}+\dot{\gamma})
\end{array}\right.
$$

Thus, for a steering angle different from zero, we are able to write a new kinematic evolution model taking into account lateral slips $\left(V_{2 x}\right.$ is deduced from the measure of an encoder mounted on the rear roll). But tests show that the resulting EKF estimator is less stable than the one presented in section 2 (in the neighbourhood of $\gamma=0$, we have used the previous model (1)). Therefore, we will consider once again that the rolls do not slip laterally and system (14) becomes [6]:

$$
\dot{\psi}=-\frac{L_{2} \dot{\gamma}+V_{1 x} \sin (\gamma)}{L_{1} \cos (\gamma)+L_{2}}
$$




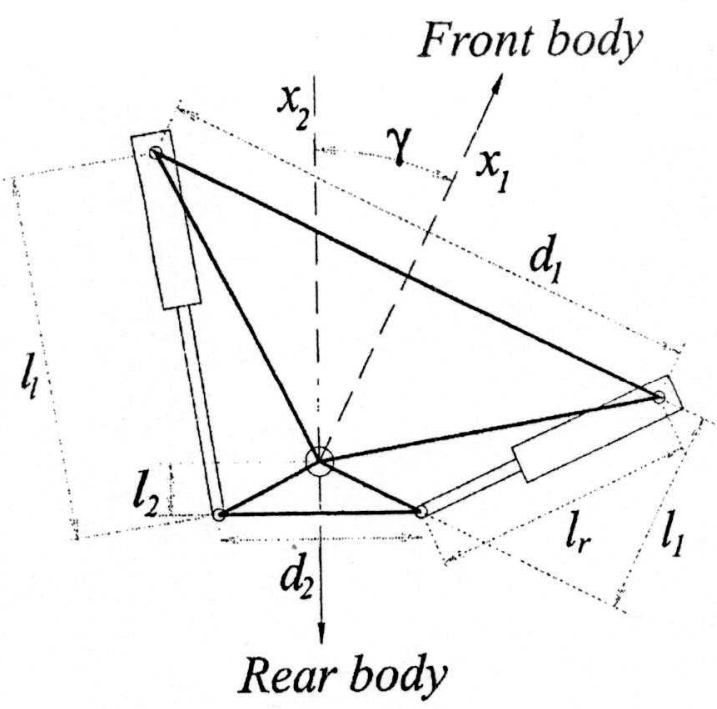

Figure 3: Steering jacks mounting

which permits us to replace the gyrometre by a steering angle measure.

To get the latter, a displacement sensor is mounted on each of both hydraulic jacks which allow the front and the rear body of the compactor to rotate relative to each other (see fig. 3). We obtain:

$$
\gamma=\arctan \left(\frac{\frac{l_{r}^{2}-l_{1}^{2}}{d_{2} l_{1}+d_{1} l_{2}}}{\frac{-\left(d_{1}^{2}+d_{2}^{2}\right)-4\left(l_{2}^{2}+l_{2}\right)^{2}+2\left(l_{l}^{2}+l_{r}^{2}\right)}{d_{1}^{2} d_{2}^{2}-4 l_{1}^{2} l_{2}^{2}}}\right)
$$

where $l_{l}$ and $l_{r}$ are the displacement sensors measures.

With this new set of sensors, the proprioceptive measures are given by:

$$
U_{i}=\left[\begin{array}{llllll}
V_{\text {radar }_{i}} & \Delta \theta_{i} & l_{l_{i}} & l_{r_{i}} & \Delta l_{l_{i}} & \Delta l_{r_{i}}
\end{array}\right]_{(17)}^{T}
$$

When $R_{1} \Delta \theta_{i}<V_{\min } T_{s}$, the elementary rotation and translation satisfy the following equations:

$$
\left\{\begin{aligned}
\Delta_{i} & =R_{1} \Delta \theta_{i} \\
\omega_{i} & =-\frac{L_{2} \Delta \gamma_{i}+R_{1} \sin \left(\gamma_{i}\right) \Delta \theta_{i}}{L_{1} \cos \left(\gamma_{i}\right)+L_{2}}
\end{aligned}\right.
$$

In the other case, we have:

$$
\left\{\begin{aligned}
\Delta_{i} & =T_{s} V_{\text {radar }_{i}}+D_{\text {radar }} \omega_{i} \\
\omega_{i} & =-\frac{L_{2} \Delta \gamma_{i}+\sin \left(\gamma_{i}\right) T_{s} V_{\text {radar }_{i}}}{L_{1} \cos \left(\gamma_{i}\right)+L_{2}+D_{\text {radar }} \sin \left(\gamma_{i}\right)}
\end{aligned}\right.
$$

To conclude with this last set of proprioceptive sensors, figure 4 shows for a given trajectory the front roll rotation speed calculated by the last two methods presented in section 3 . We find out that the gyrometre data are comparable to the ones calculated with the steering angle.
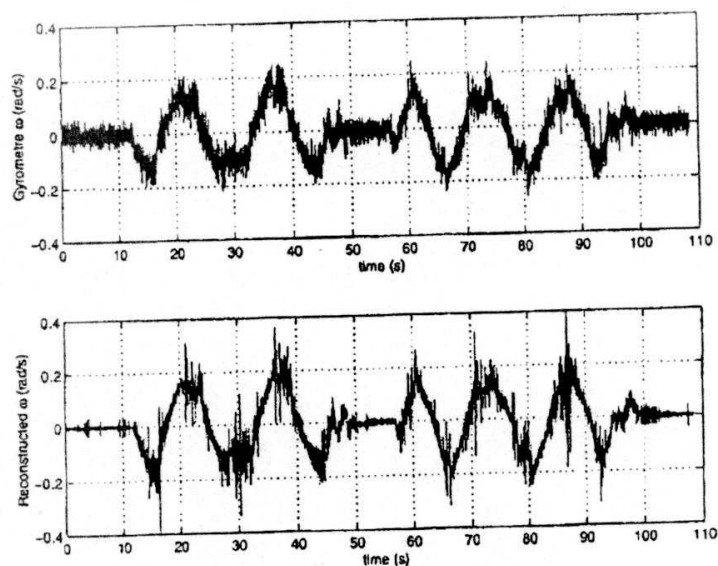

Figure 4: Comparison between measured and reconstructed front roll rotation speeds for a sinusoidal trajectory

\section{EXPERIMENTAL RESULTS}

Trials have been carried out on an Albaret road roller VA12 DV. The gyrometre is a VSG 2000 from British Aerospace, the Doppler radar is a DICKEY-john RVS II, the steering jack lengthenings are known by two magnetostrictive sensors from MTS, and the GPS receiver is a TRIMBLE 7400 MSi.

We have recorded at the sampling period $T_{s}=0.01 s$ all the signals given by the sensors mounted on the compactor and this, for different kinds of trajectories. Then, in post-processing, we have simulated GPS maskings in order to evaluate the different EKF estimator performances in sheer dead-reckoning navigation : the localisation errors are calculated by comparing the GPS measure to the estimated position at the same instant. Thus, we can check if the sets of sensors presented in section 3 are acceptable alternatives to using odometry on work sites.

For each trial, results are represented as follows:

- The first figure gives the compactor position in the work frame. Its origin is defined by the first point of the trajectory.

- The second figure represents the lateral error relative to the real trajectory made by the three Kalman estimators (mixed line for odometry, solid line for the radar + gyrometre configuration and dashed line for the radar + steering angle configuration). The vertical lines give the beginning and the end of the GPS maskings (and consequently, of the dead-reckoning navigation).

- The third figure displays the error distance between the estimated position and the real position given by RTK GPS.

Only a few trajectories are presented here. In each case, the road roller covered a distance of 
several decametres and camc back in reverse to its starting-point at the average speed of $4 \mathrm{~km} / \mathrm{h}$. In the first trial, the compactor followed a straight line. In the second one, the compactor described a curve, and in the last one, the trajectory is sinusoidal.
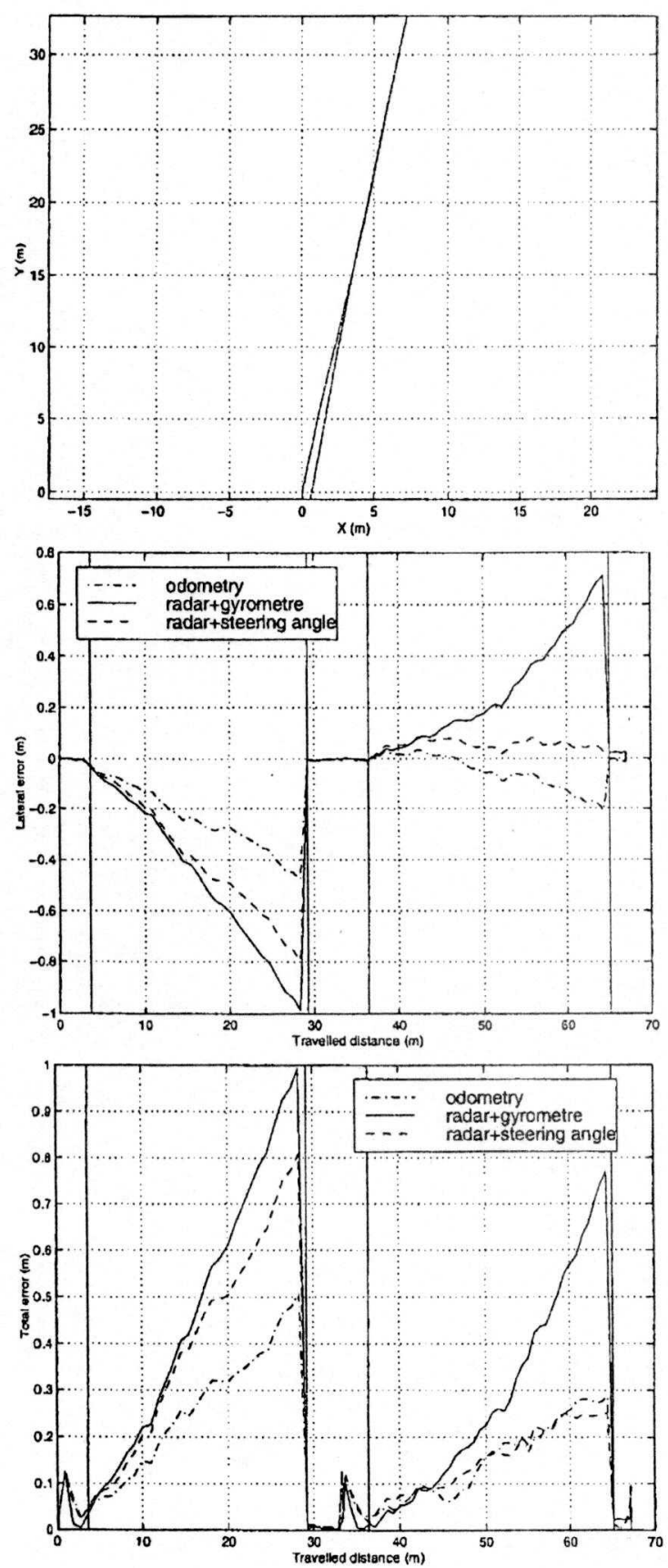

Figure 5: Straight line trajectory

The two first trials show that bicycle wheel encoders and the radar + steering angle configuration give equivalent results, with a slight advantage for odometry.

The radar + gyrometre configuration has less satisfying performances, mainly because of the drift problems. Indeed, we observed that its offset varied quickly, and we have to use each stop of the compactor to recalculate it. But if this stop is too short, the correction, when it is possible, is not sufficient, and the localisation error made during the return to the starting-point is often larger than the one made during the first part of the movement.
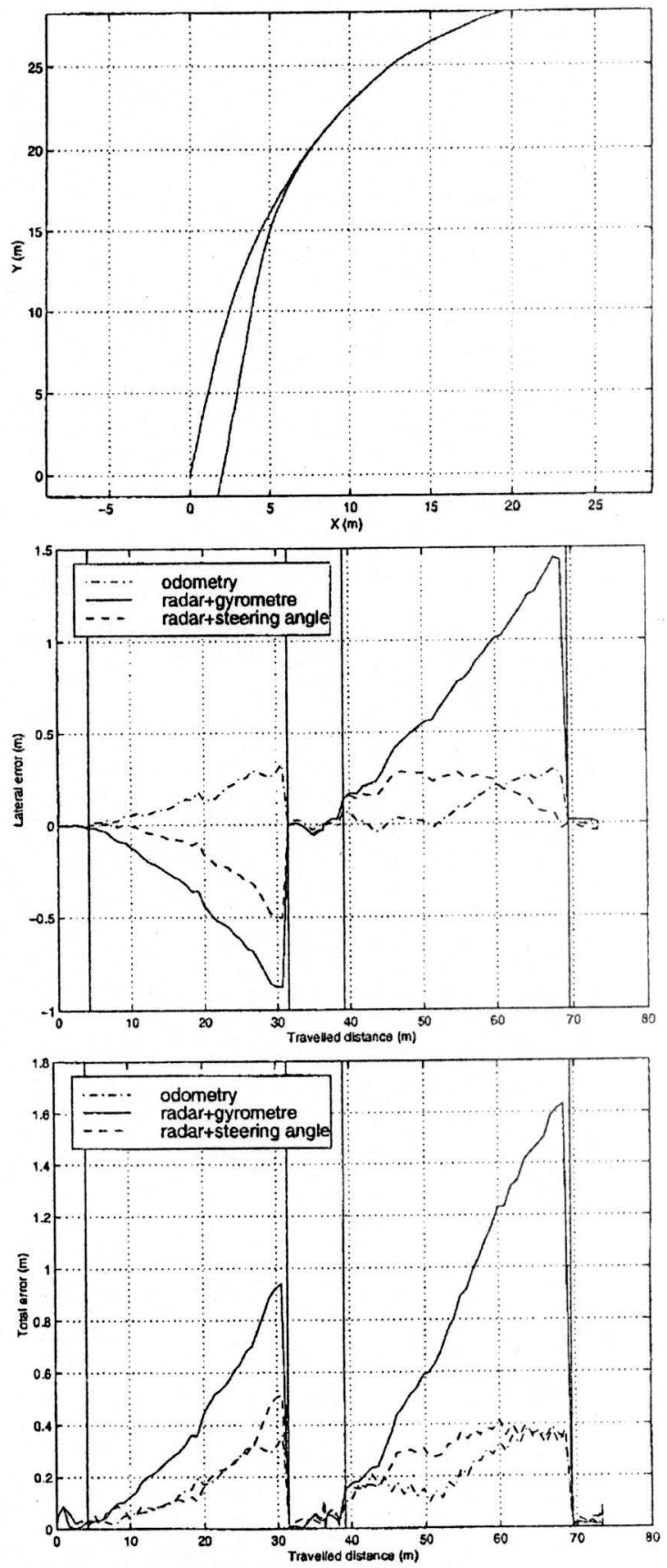

Figure 6: Curve trajectory

Eventually, the aim of trials like the third one with its sinusoidal trajectory was to solicit more dynamically the compactor steering so as to see if the reconstructed front roll rotation speed was still acceptable. Indeed, for this kind of movement, lateral slippage probably occurs, and the 
application of the equation (15) may seem dubious. Yet, results show that the radar + steering angle configuration works fairly well even in these conditions.
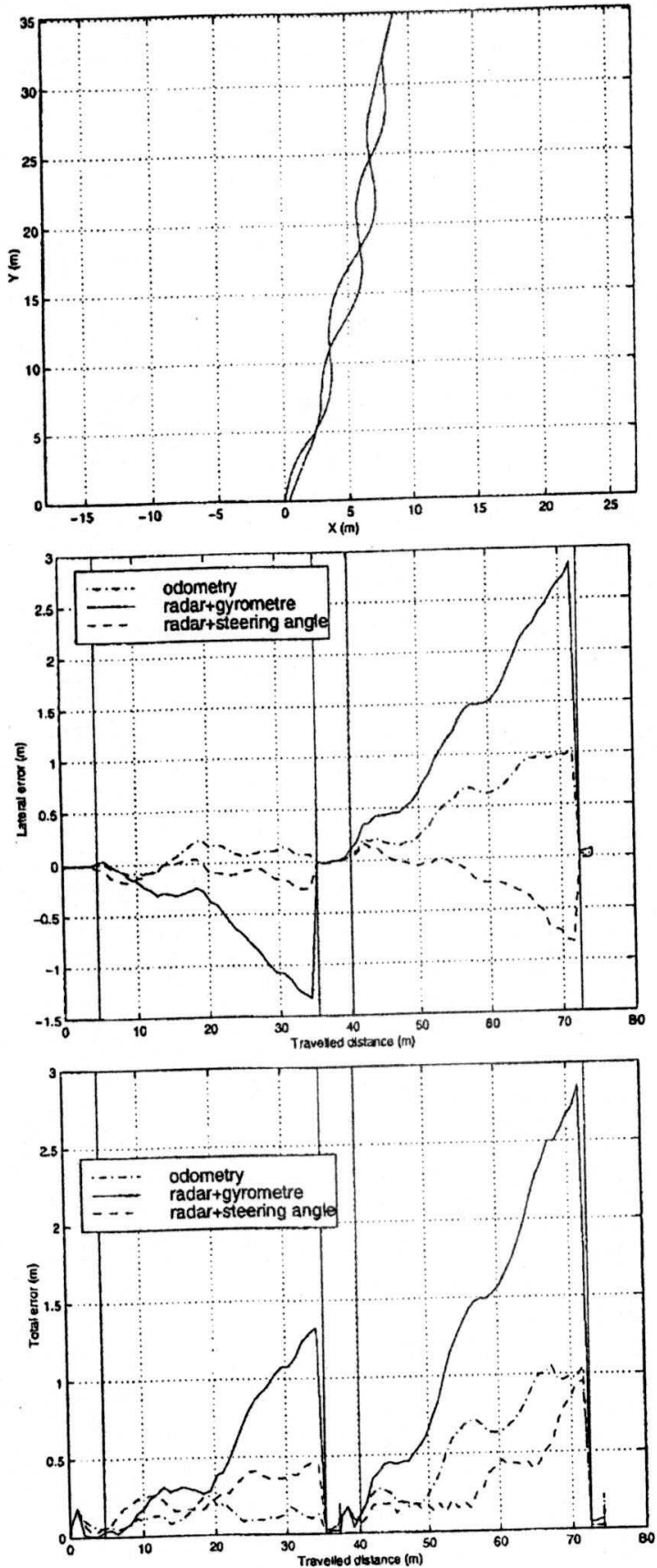

Figure 7: Sinusoidal trajectory

\section{CONCLUSIONS}

The purpose of this paper was to propose and validate alternative equipments to encoder wheels in order to implement a real-time 2D positioning system for civil-engineering machines.

Within the framework of the CIRC project, it has already been shown that by using a Kalman estimator fusing radar, fiber-optic gyrometre and RTK GPS data, satisfying results were obtained [4]. But the price of the chosen gyrometre is a major drawback when industrialization is considered.

With a cheaper instrument appear offset drift problems which are hardly compatible with the required precisions. A new solution is proposed, based on the measurement of the steering angle between the front and rear body of the compactor. The first results are encouraging, since this equipment and odomety have similar performances. Moreover, the total cost of the lengthening sensors is about 900 Euros, against 3000 to 4500 for a high-precision gyrometre, which is much more affordable for an industrial application. For all these reasons, the radar + steering angle configuration is an interesting alternative.

\section{Acknowledgments}

The authors would like to thank the LCPC and the CER for having placed the Albaret compactor to their disposal. Thanks are also due to Michel Froumentin for his inestimable help during trials.

\section{REFERENCES}

[1] B. Barshan and H.F. Durrant-Whyte. Inertial navigation systems for mobile robots. IEEE Transactions on Robotics and Automation, 11(3):328-342, 1995.

[2] P. Bonnifait, F. Peyret, and G. Garcia. New data fusion techniques for high-precision localization of civil-engineering equipment. In Proceedings of the 14th ISARC, pages 11-20, June 1997.

[3] E. Guillo, M. Gautier, and F. Boyer. Dynamic modelling and simulation of a compactor. In Proceedings of the 14th IFAC World Congress, July 1999.

[4] L.H. Pampagnin, F. Peyret, and G. Garcia. Architecture of a gps-based guiding system for road compaction. In Proceedings of the IEEE International Conference on Robotics and Automation, pages 2422-2427, May 1998.

[5] F. Peyret, D. Bétaille, and G. Hintzy. Highprecision application of gps in the field of realtime equipment positioning. In Proceedings of the 14th ISARC, pages 2-10, June 1997.

[6] S. Scheding, G. Dissanayake, E. Nebot, and H.F. Durrant-Whyte. Slip modelling and aided inertial navigation of an lhd. In Proceedings of the IEEE ICRA, pages 1904-1909, April 1997. 